\title{
¿Dónde está la utilidad?
}

\section{Alcibíades Dávila Cerro}

Dos inquietudes, una procedente de pequeños y mediamos empresarios y otra originada en el aula de clase al explicar los diferentes métodos para valuar inventarios y pedirles a los estudiantes que tomen la decisión de elegir el método que ellos consideran mas convenientes para la empresa, me llevaron a la realización de una investigación titulada Método Cuantitativo para Depurar la utilidad y en este artículo hago un bosquejo de ella.

La primera inquietud convertida en un objetivo de esta investigación es poder responder con propiedad a la pregunta de los gerentes que tienen dificultades para pagar a los proveedores y en el momento no hay disponible ni en el banco, ni en la caja pero ven en los estados financieros y escuchan de parte de los administradores financieros( Administradores, Contadores, Economistas, etc.) los buenos márgenes de utilidad que ha dejado la empresa en un período determinado ¿ donde está la utilidad?

El gerente hace la pregunta cuando tiene problemas con la liquidez y en el período se tienen unas utilidades que los financistas han considerado buena; con la pregunta deja ver un poco de escepticismo sobre lo que le están diciendo y sobre lo que está viendo en los estados financieros y no es para menos, el es conciente de los malos momentos que le está dejando la falta de dinero y ahora se le suma que la utilidad debe ser distribuida una parte para el gobierno(35\%) y la otra para la empresa $(65 \%)$ de la última parte el sabe que por lo menos el $50 \%$ debe ser repartido a los socios ; esto aumentará la iliquidez y los problemas de la empresa .

Los gerentes están al tanto de lo que sucede diariamente con el efectivo de la empresa, ya que todos los días reciben y evalúan los informes sobre ese con- 
cepto, por eso cuando la utilidad es alta ( buena según la inversión) pero la liquidez es mala, tiende a existir en sus mentes dudas sobre esa utilidad.

Nuestro trabajo de investigación tiende a dar respuesta a los gerentes con esas inquietudes y a profundizar más sobre el concepto de la utilidad: utilidad bruta en venta, utilidad en operación, etc, pero teniendo como tema central el determinar un método que nos permita evaluar la calidad de la utilidad obtenida

\section{FUNDAMENTOS APLICADOS}

Los fundamentos contables y financieros utilizados en ésta investigación son los siguientes:

1. Partida Doble

2. Causación

3. Ciclo Contable

4. Estados Financieros

4.1 Balance General

4.2 Estado de Resultado

4.3 Estado de Usos y Fuentes de Fondo

4.4 Flujo de Efectivo

5. Generación Interna de Fondo

Tratamos de probar que la utilidad tiene una calidad; ésta como es lógico se debe reflejar en los activos pero no siempre estos contribuirán al desarrollo de la empresa

Por ejemplo: Si una empresa tiene en un período contable, una utilidad de 50 millones de pesos (cifra que se considera excelente para la inversión realizada) y esta utilidad se encuentra reflejada en la cuenta de banco, podemos decir que esa empresa en ese período ha obtenido un excelente rendimiento financiero y una excelente calidad de la utilidad, a la cual por estar completamente líquida le damos un puntaje de 100 ( puntaje máximo). ¿ Podríamos decir lo mismo si los 50 millones están reflejados en un terreno, mal ubicado para los actuales mo- 
mentos económicos, y si la empresa saliera a venderlo le costaría trabajo realizarlo y solo lo lograría dándolo por un precio muy inferior al valor en libro?

En ambas situaciones la utilidad es 50 millones y ese rendimiento financieramente es considerado excelente pero la calidad de la utilidad no es igual; mientras en el primer caso la calidad se considera inmejorable, ya que está totalmente líquida en el segundo caso la calidad de la utilidad es pésima y tendrá repercusiones muy negativas para la empresa. Que podríamos decir si la utilidad de esa empresa está reflejada en inventarios obsoletos, que nadie quiere comprar, o en una cartera excesivamente morosa, cuyos clientes difícilmente le pagarán a la empresa, en estos casos la utilidad que presenta la empresa no es real.

Con este trabajo queremos lograr el diseño de un método que nos permita medir la calidad de la utilidad de una empresa en un período determinado.

Partimos con el supuesto que el análisis de la utilidad requiere tener para su validez de dos componentes:

1) El que tiene que ver con las razones financieras, utilidad sobre venta; utilidad sobre la inversión de los socios; utilidad operacional y en general con todas aquellas razones financieras que nos permitan realizar una buena evaluación de la utilidad obtenida y hacer su comparación con los márgenes que el mercado acepta como buenos en esos momentos.

2) Determinar el grado de calidad de esa utilidad.

Casi todos los libros de finanzas tratan con profundidad el primer punto, pero casi ninguno ${ }^{1}$. trata el segundo, el cual es considerado como esencial y determinante para la aceptación de la utilidad como tal.

Ejemplo: una empresa considera como optima la utilidad que deje un $50 \%$ sobre la inversión de los socios; la empresa tiene invertido 40 millones y en un

${ }^{1}$ No conocemos libros de Finanzas que traten este tema, pero no podemos afirmar que ninguno lo toque. 
período se gana 25 millones; está situación deja un margen de utilidad sobre la inversión de los socios del 62,5\%, margen superior en un 12,5\% al optimo esperado; sin embargo, y a pesar que las cifras presentadas son muy alentadoras, consideramos que es necesario hacerle un análisis a la calidad de la utilidad antes de aceptar que en ese período se ha obtenido un excelente resultado.

A continuación le proponemos, estimado lector, para que decida en cual de las 3 empresas de las que mostramos sus estados de resultados invertiría usted, considere que ellas están en una situación ceteris paribus,

\section{ESTADO DE RESULTADO}

3 EMPRESAS. LTDA

(EN MILLONES) Año 2002

\begin{tabular}{|c|c|c|c|c|c|c|}
\hline & & TEX & & SULFI & & LIMBO \\
\hline Ventas & & 600 & & 600 & & 600 \\
\hline \multicolumn{7}{|l|}{ - C.M.V. } \\
\hline Inventario inicial & 150 & & 150 & & 150 & \\
\hline + Compras & $\underline{370}$ & & $\underline{370}$ & & $\underline{370}$ & \\
\hline C.M.D.P.L.V. & $\overline{520}$ & & $\overline{520}$ & & $\overline{520}$ & \\
\hline - Inventario final & $\underline{120}$ & & $\underline{156}$ & & $\underline{90}$ & \\
\hline C.M.V & & 400 & & 364 & & 430 \\
\hline U.B.V. & & 200 & & 236 & & 170 \\
\hline Gastos de Operación & & 100 & & 100 & & $\underline{100}$ \\
\hline Utilidad de Operación & & 100 & & 136 & & 70 \\
\hline -Imporrenta & & $\underline{35}$ & & $\underline{47,6}$ & & 24,5 \\
\hline Utilidad Neta & & 65 & & 88,4 & & 45,5 \\
\hline Participaciones & - & $\underline{32,5}$ & & 44,2 & & 22,75 \\
\hline Ganancias acumuladas & & 32,5 & & 44,2 & & 22,75 \\
\hline
\end{tabular}


Quizás usted, al igual que la mayoría de las personas a las cuales se les hace esta pregunta responderá que en la empresa Sulfi, dado que si las condiciones financieras, organizativas, de mercado y de proyección a largo plazo son iguales para estas tres empresas, es de suponer que usted deba invertir en la empresa que más utilidad está dejando y por ende mayor participación. Sin embargo en el ejercicio que hacemos en la clase de contabilidad, no se trata de tres empresas sino de una sola y aquí la decisión que hay que tomar es que método de inventario le conviene más a la empresa; después de unos sencillos cálculos aritméticos para darle valor al inventario el estado de resultado nos queda de la siguiente manera:

\section{ESTADO DE RESULTADO EMPRESA TEXTILES DEL CARIBE (EN MILLONES) Enero-Dic año 2002}

\begin{tabular}{|c|c|c|c|c|c|c|}
\hline & & P.P & & P.E.P.S & & U.E.P.S \\
\hline Ventas & & 600 & & 600 & & 600 \\
\hline \multicolumn{7}{|l|}{ - C.M.V. } \\
\hline Inventario inicial & 150 & & 150 & & 150 & \\
\hline + Compras & 370 & & 370 & & 370 & \\
\hline C.M.D.P.L.V. & $\overline{520}$ & & $\overline{520}$ & & 520 & \\
\hline - Inventario final & 120 & & 156 & & 90 & \\
\hline C.M.V & & 400 & & 364 & & 430 \\
\hline U.B.V. & & $\overline{200}$ & & $\overline{236}$ & & $\overline{170}$ \\
\hline Gastos de Operación & & 100 & & 100 & & $\underline{100}$ \\
\hline Utilidad de Operación & & 100 & & $\overline{136}$ & & 70 \\
\hline -Imporrenta & & $\underline{35}$ & & $\underline{47,6}$ & & 24,5 \\
\hline Utilidad Neta & & 65 & & 88,4 & & 45,5 \\
\hline Participaciones & & $\underline{32,5}$ & & $\underline{44,2}$ & & 22,75 \\
\hline Ganancias acumuladas & & 32,5 & & $\overline{44,2}$ & & $\overline{22,75}$ \\
\hline
\end{tabular}

Aquí las respuestas no son tan abrumadoras por el P.E.P.S (primera en entrar primera en salir) pero la mayoría escoge este método, con el argumento que utilidad es utilidad y donde mayor sea esta partida hay debemos apuntar y esto porque tienen la idea que la utilidad está reflejada en la caja, o sea, que en el 
caso que analizamos al escoger el P.E.P.S se supone que en la caja hay 88,4 millones y si se escoge P.P (promedio ponderado) tendremos solamente 65 millones, cosa que es totalmente equivocada; algunos pocos escogen el U.E.P.S ${ }^{2}$ (último en entrar primero en salir) con el argumento que así se le paga menos impuestos al gobierno y menos participaciones a los socios, de esta manera la empresa tendrá mas recursos para encarar el nuevo período y producir mas utilidades en el futuro; en algunos casos uno que otro estudiante se muestra decepcionado de la contabilidad y pregunta porqué los tres métodos no dan iguales si se trata de la misma empresa y el resultado debería ser uno solo y de no ser así entonces la utilidad puede ser manipulada, como efectivamente así sucede.

Todo lo anterior me hace pensar que la utilidad tiene una calidad y por lo tanto se hace necesario conocer un método que nos permita determinar en que cuentas del activo está reflejada la utilidad de un período para conocer que tanta calidad tiene ésta, lo cual nos servirá para tomar decisiones tendientes a una mejor y más eficiente proyección en nuestra empresa como también para señalar los posibles errores cometidos en un período, los cuales deben ser corregidos en el siguiente.

Para adelantar el proceso de determinar la calidad de la utilidad se requiere de dos balances de períodos consecutivos y del último estado de resultado; con ellos haremos estado de Usos y Fuentes, estado de flujo de efectivo, se determinará la Generación Interna de Fondo y se define en que cuentas se refleja la utilidad, luego se le da un puntaje ( en una escala de 0 a 100 puntos) y de acuerdo al número de puntos obtenidos se define si la calidad es excelente, buena, regular o mala

Para la total comprensión del Método Cuantitativo para Depurar la Utilidad, la Facultad de Ciencias Económicas de la Universidad de Cartagena, ofrece a las persona interesadas un seminario el cual tiene una duración de 6 horas y se puede dictar los fines de semana en las instalaciones de la Universidad o en la sede de las empresas que lo deseen en el tiempo convenido por las partes.

${ }^{2}$ En Colombia la legislación tributaria prohíbe la utilización del método U.E.PS. 